\title{
Estudio del comportamiento térmico de agua para uso residencial en tinacos de concreto y polietileno en un clima cálido-subhúmedo
}

\author{
Study of the Thermal Behaviour of Water for Residential Use in Tanks \\ of Concrete and Polyethylene in Humid Subtropical Climate
}

\author{
Diego-Ayala Ulises \\ Centro de Investigación Científica de Yucatán AC (CICY) \\ Departamento de energía renovable \\ Correo:udiego@outlook.com
}

\author{
Carrillo-Baeza José Gonzalo \\ Centro de Investigación Científica de Yucatán AC (CICY) \\ Departamento de materiales \\ Correo:jgcb@cicy.mx
}

Información del artículo: recibido: noviembre de 2014, aceptado: diciembre de 2014

\section{Resumen}

Este artículo presenta un estudio comparativo del comportamiento térmico de dos tinacos residenciales, de polietileno y concreto, expuestos al sol a lo largo de un año en el estado de Yucatán. Se midieron la irradiación solar y las temperaturas correspondientes alcanzadas por cada sistema. Se identificaron los patrones diarios de elevación y reducción de temperatura y se determinó la cantidad de energía adquirida durante el día y el calor disipado durante la noche, a fin de definir la posibilidad de la utilización de tinacos de agua residenciales como fuente de agua caliente en casas habitación en la región de Yucatán. Con este estudio se ha determinado que los períodos del día en que se puede contar con agua caliente a la temperatura de confort para ducharse son limitados y que, de forma interesante, ambos sistemas entregan temperaturas similares en el fondo de los tanques a lo largo del año.

\footnotetext{
Abstract

This article presents a comparative study of the thermal behavior of residential water tanks of polyethylene and concrete exposed to the sun over a year in the state of Yucatan. The energy for radiation and their corresponding temperatures in each system were measured. Daily patterns of elevation and reduction of temperature were identified and the amount of energy acquired during the day as well as the heat dissipated overnight were determined, aiming to determine the possibility of using residential water tanks as a source of hot water in residential homes in the Yucatan region. Based on this study it has been found that the periods of the day with hot water temperature for showering with comfort is limited and that, interestingly, both systems show similar temperatures at the bottom of the tanks throughout the year.
}

\section{Descriptores:}

- energía solar

- depósito residencial

- comportamiento térmico

- estudio anual

- tinaco

- polietileno

- concreto

\section{Keywords:}

- solar energy

- residential tank

- thermal behavior

- annual study

- water tank

- polyethylene

- concrete 


\section{Introducción}

El estado de Yucatán se conoce por ser un lugar típicamente caluroso a lo largo del año, teniendo comúnmente temperaturas promedio máximas en un día entre 30 y $35^{\circ} \mathrm{C}$. Dichas temperaturas son consecuencia de la irradiación que llega a la zona, que fluctúa entre 6.19 y $3.61 \mathrm{kWh} / \mathrm{m}^{2} /$ día (Barron, 2012). Estas condiciones favorecen la instalación de sistemas solares para el aprovechamiento de la energía renovable del sol. Una aplicación viable para su aprovechamiento es utilizar calentadores solares residenciales de agua. Sin embargo, se tiene la incógnita respecto a la necesidad de la instalación de estos equipos, pues debido a la sensación de calor predominante en Yucatán se tiene la creencia que un calentador de agua puede incluso no ser necesario. Sin embargo, dicha creencia queda en entredicho al observar que según el Instituto Nacional de Estadística y Geografía (INEGI) en el estado de Yucatán, teniendo una media nacional de $47.83 \%$ en todo el país, $19.61 \%$ de las viviendas particulares habitadas disponían de algún sistema para el calentamiento de agua (como boiler de gas o eléctrico) en 2010 (INEGI, 2010).

Casi todos los estudios en los que se incluyen los hábitos de una ducha caliente, como los realizados por Emery y Kippenhan (2004) y Guerra et al. (2009), están enfocados al análisis de la cantidad de energía utilizada de manera cotidiana para todas las necesidades de la vivienda, que incluye el calentamiento de agua para uso doméstico, aunque cabe señalar que en estos casos es una condición requerida, particularmente al ser estudios hechos en países que tienen temperaturas, en especial en invierno, mucho más bajas que en la región de Yucatán. En el estado del arte se observa que hay muy pocos estudios enfocados a las condiciones de temperatura del agua para su uso en duchas (aseo corporal), donde lo más cercano lo presenta Kordana et al. (2014), enfocado en la utilización de sistemas para ahorrar energía a partir del agua utilizada para ducharse. Si bien, se estudian maneras de optimizar la energía utilizada durante una ducha, se tiene como requerimiento mantener temperaturas adecuadas para un baño confortable, pues como indican Boduch y Fincher (2009), los seres humanos somos sensibles a pequeñas diferencias en la temperatura.
A la fecha, no se han identificado estudios relacionados con el comportamiento térmico de los depósitos de agua residenciales; por ello, para dar respuesta a esta incógnita, se llevó a cabo un estudio de la evolución térmica del agua en tinacos residenciales en la región de Yucatán a lo largo de un año.

\section{Desarrollo}

\section{Materiales y métodos}

\section{Depósitos de agua}

Se llevó a cabo el estudio con dos depósitos de agua tipo residencial (de interés social), ya que son los más comunes en la región: uno de polietileno de alta densidad de 450 litros (color beige) y otro de concreto de 400 litros (color natural), los cuales fueron aforados a 360 litros (con 0.62 y $0.96 \mathrm{~m}$ de altura de columna de agua para el de polietileno y el de concreto, respectivamente) y colocados sobre techo en un área libre de obstáculos, teniendo entre centros una distancia lineal de 5 metros.

Se instaló en ambos depósitos una conexión de agua de entrada mediante válvula y flotador de manera que automáticamente se mantuvieran llenos, compensado las posibles pérdidas por evaporación. Ninguno de estos dos tinacos proporcionó servicio de agua durante el período de estudio, por lo cual no había afectación en la temperatura por renovación de agua, midiendo así únicamente las variaciones de temperaturas generadas por calentamiento y enfriamiento por influencia de la ra-

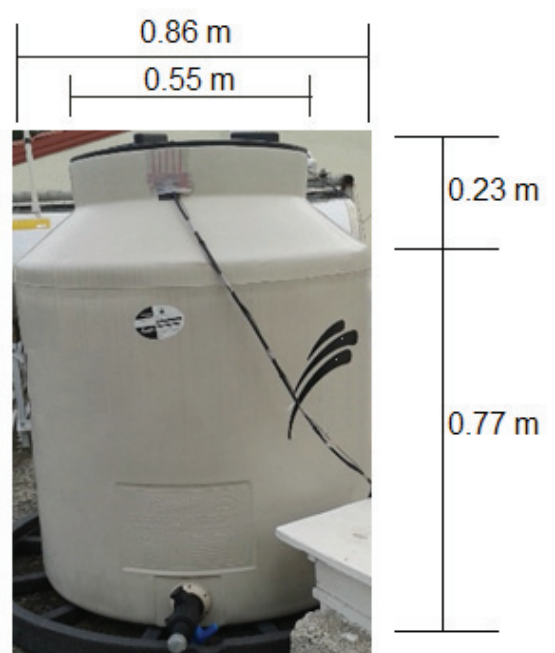

a)

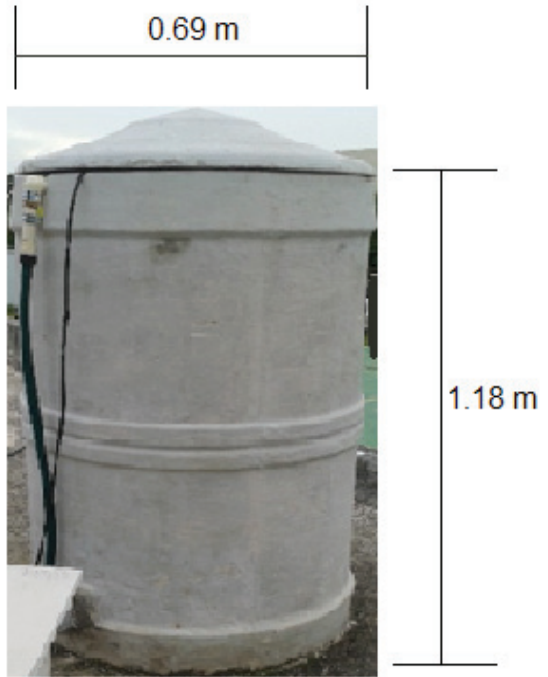

b)
Figura 1. Fotografías y dimensiones internas de los depósitos de agua instalados: a) de polietileno (espesor de $0.008 \mathrm{~m}$ ) y b) de concreto (espesor de $0.038 \mathrm{~m}$ ) 
diación y el medio ambiente durante los períodos diurnos y nocturnos. La figura 1 presenta fotografías de los depósitos de agua estudiados.

\section{Instrumentación}

Para obtener el perfil de calentamiento del agua se llevó a cabo la instrumentación de cuatro termopares tipo $\mathrm{K}$ (calibre 30 con forro de fluoro-etilen-propileno, FEP), en cada uno de los tinacos, colocados a cuatro diferentes niveles de profundidad: superficie, 2/3, 1/3 y fondo de cada tinaco. Cada conjunto de termopares se conectó a un sistema de datalogger Omega HH309A, digitalizando las mediciones con una frecuencia de adquisición de datos de una medición por minuto $(0.017 \mathrm{~Hz})$. Se realizaron tres calibraciones de los dispositivos termopares a lo largo del estudio; al inicio, a los 6 meses y al final del estudio, cuidando correspondencia en todo momento.

\section{Mediciones de irradiancia y medio ambiente}

Para el estudio se obtuvieron datos precisos de la irradiancia y temperatura ambiente mediante una estación meteorológica marca Davis Pro2 Plus ubicada en la ciudad de Mérida, a $21^{\circ} 01^{\prime} 47.8^{\prime \prime}$ latitud Norte y 89³8'17.0' longitud Oeste, en la península de Yucatán. La estación se encuentra instalada a 12 metros sobre el nivel del mar en un espacio libre de obstáculos.

\section{Período de mediciones}

Se llevaron a cabo mediciones continuas de las temperaturas en los depósitos de agua durante un año, de septiembre del 2013 a agosto de 2014.

\section{Discusión y análisis de resultados}

\section{Irradiación solar y temperaturas}

Durante el período de estudio se registraron valores de irradiancia a lo largo del día, con lo que se estimó la irradiación solar, calculándose entre 1 y $7 \mathrm{kWh} / \mathrm{m}^{2} /$ día, graficado en la figura 2. Como es de esperarse, la cantidad de irradiación solar incidente disminuye para el período comprendido de entre noviembre y febrero. La dispersión observada corresponde a datos típicos climáticos con variables combinadas de radiación, nubosidad y ángulo solar a lo largo de un año.

Respecto a las temperaturas ambiente mostradas en la figura 3, se registraron valores promedio de entre 21 y $33^{\circ} \mathrm{C}$ diario, observándose un patrón de respuesta correspondiente a la irradiación solar.
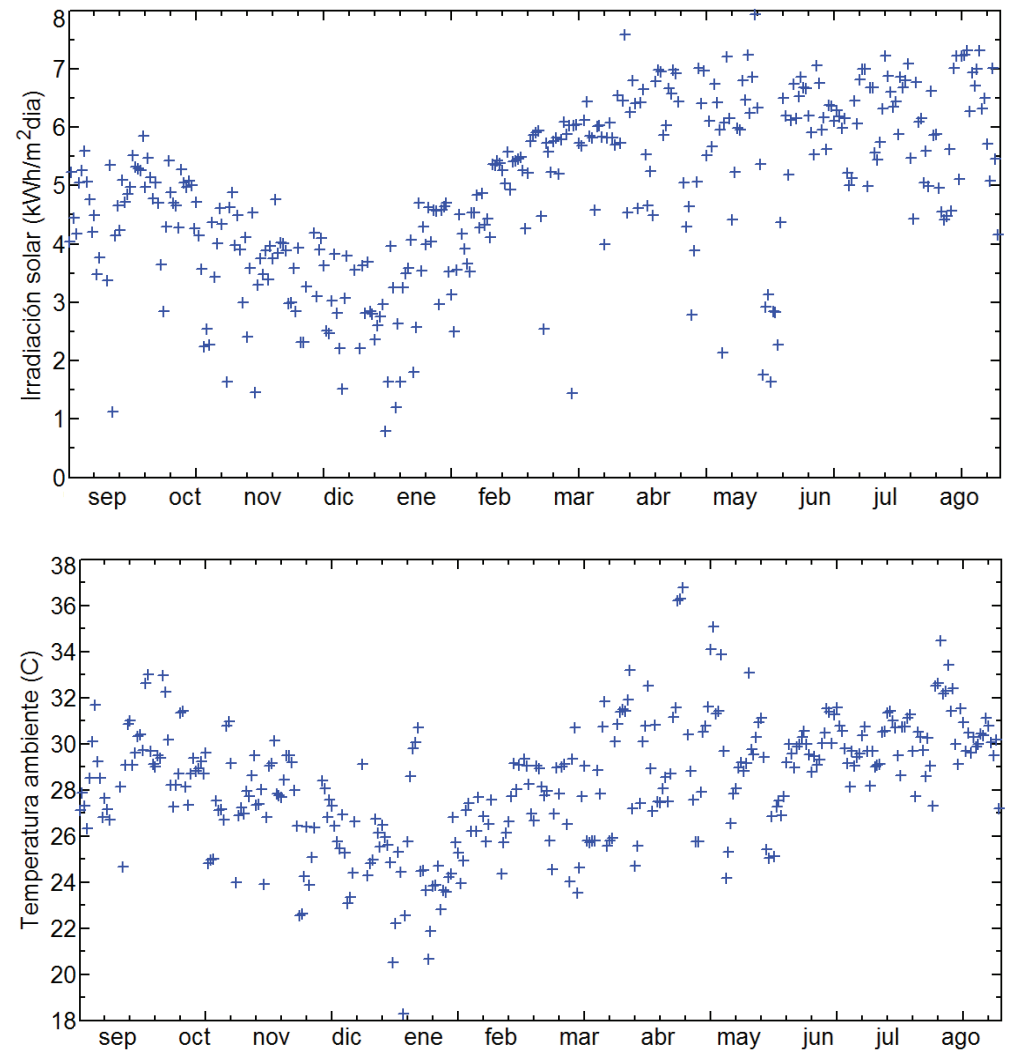

Figura 2. Irradiación solar diaria en el período septiembre 2013-agosto 2014

Figura 3. Temperaturas ambiente promedio diaria en el período de septiembre 2013-agosto 2014 


\section{Patrón de temperaturas}

La figura 4 presenta el comportamiento típico del depósito de concreto a lo largo de un día (en este caso para el 30 de julio del 2014). Se puede observar que durante la noche las temperaturas tienden a igualarse y durante el día se separan. A lo largo del día, mientras se recibe la radiación del sol, el nivel superior tiende a separarse en mayor proporción que las otras mediciones, las cuales tienden a mantenerse equidistantes. Durante la noche, las temperaturas tienden a igualarse, observándose la temperatura del fondo ligeramente separada del resto del grupo en todo momento. En este día, la temperatura máxima del sensor del fondo, reporta temperaturas de alrededor de $31^{\circ} \mathrm{C}$, con un nivel de irradiancia máxima de $920 \mathrm{~W} / \mathrm{m}^{2}$, correspondiente a un día soleado. La temperatura del fondo es de particular interés debido a que es el agua de la que se dispone en uso residencial, con el arreglo típico de tubería tradicional disponible. Cabe resaltar, que si uno quisiera disponer de agua más caliente, esto sería posible por medio de un dispositivo que pudiera extraer el agua cercana a la superficie cuya temperatura, para este caso, es de hasta $34^{\circ} \mathrm{C}$.

La figura 5 presenta el comportamiento térmico típico del depósito de polietileno a lo largo del mismo día estudiado. Durante las primeras horas de la madrugada y durante el día se aprecia un comportamiento similar entre las temperaturas, con los sensores del depósito
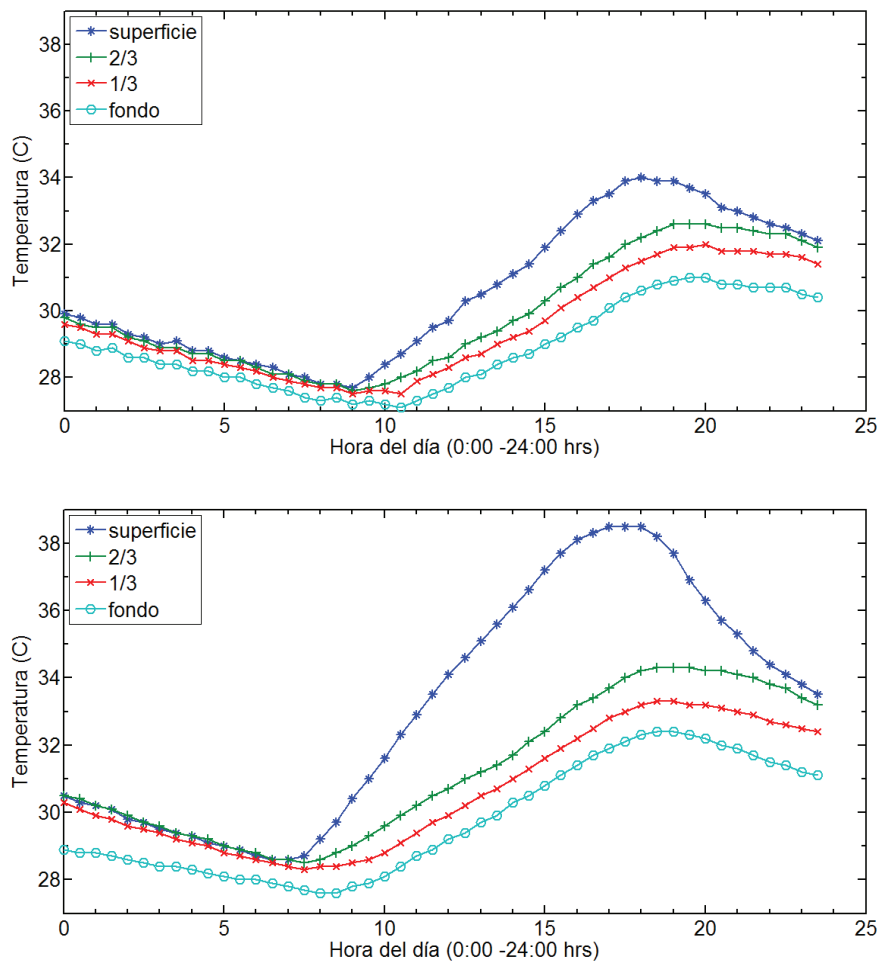

de concreto. Aunque el comportamiento es similar en ambos depósitos, durante el día es particularmente diferente en el nivel superior, en el que se aprecia un incremento mayor de temperatura con respecto a los otros tres niveles. Se estima que ocurre dicho calentamiento debido al efecto invernadero de la parte superior del depósito que contiene aire caliente, ya que la parte correspondiente superior de este depósito está expuesta al sol. Al caer la noche, el patrón de comportamiento vuelve a ser muy similar en todos los niveles en ambos depósitos, reflejo de la pérdida de calor al exterior en ausencia del sol. En general, los patrones de comportamiento entre un depósito y otro son iguales, donde igualmente, la temperatura del fondo se mantiene separada del resto a todo momento. Para este día comparativo (soleado), se contó con una temperatura de fondo de alrededor de $32^{\circ} \mathrm{C}$, pudiendo contar con agua más caliente en la superficie (alrededor de $38^{\circ} \mathrm{C}$ ).

La irradiancia y temperatura ambiente reportadas para ese día se muestran en la figura 6. Estas gráficas sugieren que la temperatura del agua en el depósito aumenta conforme recibe la radiación disponible a través del cuerpo del contenedor, mostrando los valores máximos de temperatura en los depósitos alrededor de las 19:00 horas, justo antes de terminar la presencia de la radiación solar. La energía alcanzada por radiación para este día es $6.76 \mathrm{kWh} / \mathrm{m}^{2} /$ día y la máxima temperatura ambiente $35^{\circ} \mathrm{C}$.

Figura 4. Comportamiento térmico típico del depósito de concreto en un día

Figura 5. Comportamiento térmico típico del depósito de polietileno en un día 
Es importante resaltar que el agua de la que típicamente se dispone en forma inmediata (por tener la descarga en la parte inferior del depósito) es la correspondiente al sensor del fondo, siendo para este caso, como se aprecia en la figura 4 , valores mínimo y máximo reportados durante el día de 27 y $31^{\circ} \mathrm{C}$, a las 8 y a las 19 horas, respectivamente.

Con fines comparativos, la figura 7 presenta una gráfica de las mediciones de irradiancia (con un total de irradiación solar de $4.5 \mathrm{kWh} / \mathrm{m}^{2} /$ día) con temperatura ambiente y de la temperatura de los dos depósitos en un día soleado típico del mes de enero (25 de enero del 2014), donde se observa que el patrón de comportamiento térmico del agua es consistente con lo mostrado en julio; observándose que el depósito de polietileno alcanza la temperatura mayor en la superficie (de ambos sistemas) y una temperatura similar en el fondo de los contenedores.

\section{Temperaturas máximas}

Una parte importante del estudio es la determinación de la temperatura existente en el agua contenida en los depósitos en diferentes estratos térmicos. El promedio mensual de las temperaturas máximas en superficie y fondo se calcula identificando el valor más alto medido a lo largo de cada día y promediándolo para cada mes. Al graficar estos valores (figura 8), se aprecia que la di-
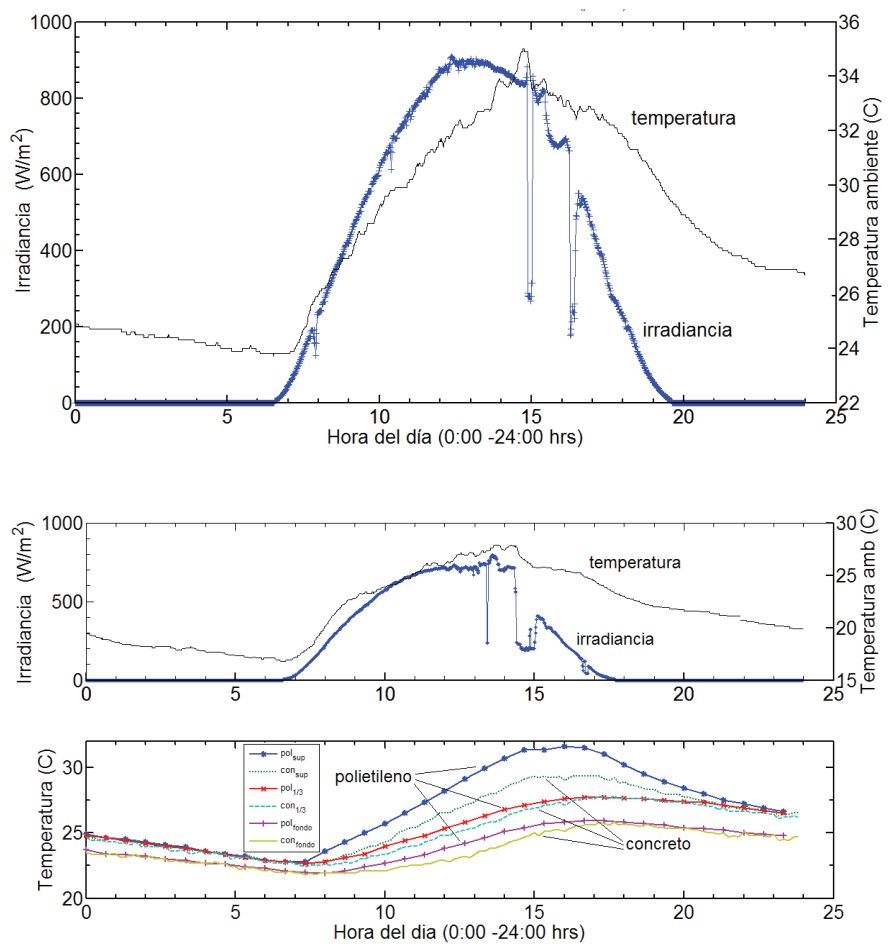

ferencia de temperaturas entre superficie y fondo tiende a ser de 6 grados para el depósito de polietileno. Por otro lado, para el caso del depósito de concreto, la diferencia es de 2 grados, entreviéndose que el flujo de calor es menor en el depósito de concreto a lo largo del año. Igualmente se puede observar que el mes que reporta la más alta temperatura corresponde al mes de julio para ambos contenedores. Es interesante que pese a las diferencias de temperaturas observadas, los valores correspondientes a los fondos de los contenedores, se aprecian similares durante todo el año.

Este estudio busca cuantificar la disponibilidad de agua a determinada temperatura que se pueda considerar confortable en la ducha para el cuerpo humano, valor que es relativo a lo que cada individuo pueda considerar como frío o caliente, relativo igualmente a la temperatura y humedad ambiental, que puede imprimir modificaciones en la sensación térmica de cada uno.

En la figura 9 se presenta la media y las desviaciones estándar de los valores presentados a las horas habituales de ducharse, consideradas en este estudio a las 7:00 y a las 20:00 horas, junto con los máximos presentados en cada día, que tienden a ocurrir entre las 17:00 y las 18:00 horas. El depósito de polietileno presenta los valores de temperatura promedio más altos $\left(31^{\circ} \mathrm{C}\right)$, con un ligero descenso para las 20:00 horas, observando que típicamente, al día siguiente la temperatura desciende de 29 a $27^{\circ} \mathrm{C}$. De forma similar, el contenedor de

Figura 6. Irradiancia y temperatura ambiente registrada en un día

Figura 7. Irradiancia y comportamiento típico, mostrando superficie, 1/3 de columna de agua y fondo, de los dos depósitos 

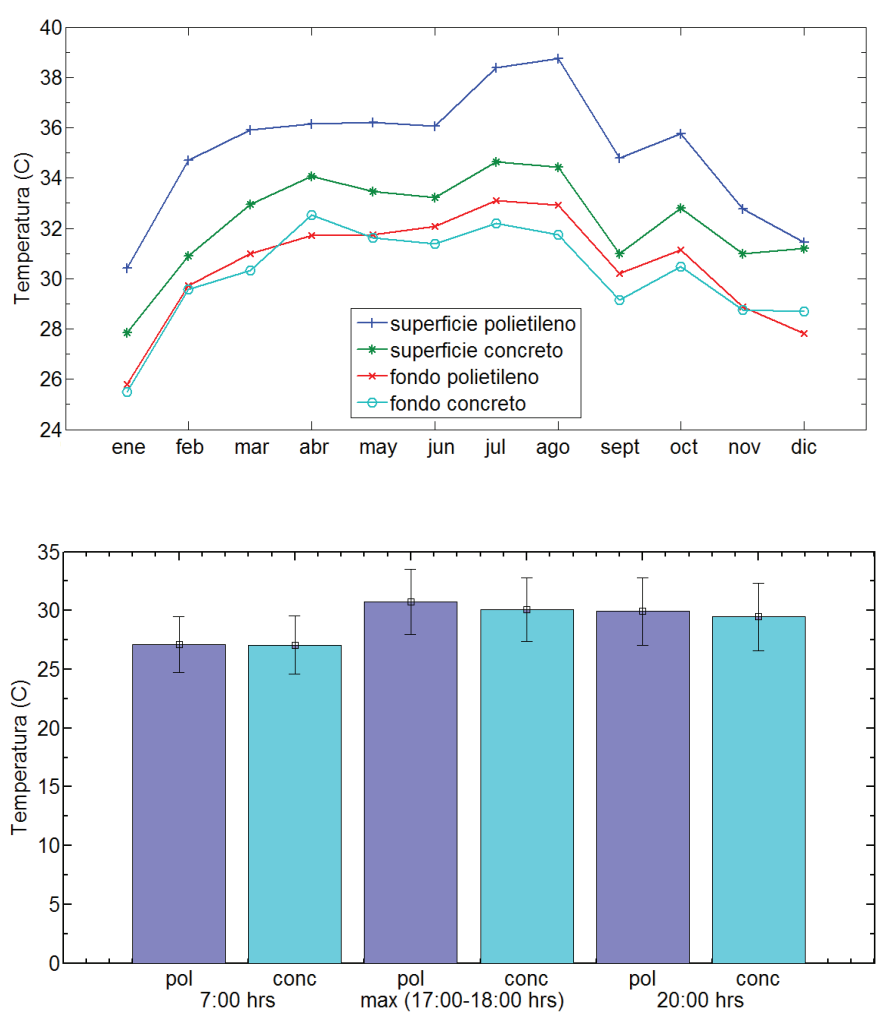

Figura 8. Promedio mensual de temperaturas máximas en la superficie y el fondo de los tinacos
Figura 9. Promedio de temperaturas en el fondo de los depósitos de polietileno (pol) y concreto (conc) durante períodos de mañana (7 horas), noche (20 horas) y de máximas temperaturas (max (17:00-18:00 horas) concreto alcanza su temperatura promedio máxima de alrededor de $29^{\circ} \mathrm{C}$, bajando aproximadamente un grado para la hora de la ducha, a las 20:00 horas. Las temperaturas promedio de la mañana muestran valores similares al contenedor de polietileno, oscilando alrededor de los $27^{\circ} \mathrm{C}$.

La norma oficial mexicana NOM-022-SCFI-1993, referente a calentadores instantáneos de agua para uso doméstico, indica que después de 15 minutos de funcionamiento a plena capacidad la temperatura máxima permitida en la perilla o manija de control es $50^{\circ} \mathrm{C}$. Análogamente, la Thermostatic Mixing Valve Manufacturers Association del Reino Unido indica que los objetivos para cualquier sistema de control de agua son almacenarla a $60^{\circ} \mathrm{C}$, distribuirla a $55-60^{\circ} \mathrm{C}$ y entregarla a temperaturas de descarga de entre $35-46^{\circ} \mathrm{C}$ ( TMVMA, 2000). En línea con estos valores y considerando la temperatura corporal promedio de $36.5^{\circ} \mathrm{C}$ como temperatura de confort, dado que sensaciones de incomodidad en el cuerpo humano están asociadas con cambios a partir de esta temperatura (Gagge et al., 1967), únicamente algunos valores extremos podrían calificar para niveles confortables de temperatura, donde en promedio, tanto el contenedor de concreto como el de polietileno ofrecen temperaturas similares. Cabe resaltar también, que cuando estos sistemas están en uso en una residencia, la válvula de llenado se encarga de completar el volumen usado, donde el agua de reposición es típicamente más fría por provenir de tubería subterránea.

Aunque el valor promedio es muy similar en la noche en ambos depósitos, una inspección detallada de la distribución de la temperatura en el fondo a las 20:00 horas revela que la mayor frecuencia de temperaturas altas se tiene en el depósito de polietileno, (figura 10a), donde sumando las frecuencias de ocurrencias entre 32 y $36^{\circ} \mathrm{C}$, se obtiene $48 \%$, mientras que en el de concreto (figura 10b) en el mismo intervalo corresponde a $32 \%$ de ocurrencias. Por tanto, añadiendo el efecto de la mayor temperatura en las capas superiores, es claro que durante el día y hasta la noche el depósito de polietileno tendrá agua más caliente que el de concreto, aunque sin alcanzar temperaturas de confort.

Si se incluyen los efectos de pérdida de calor durante el trayecto en la tubería y en la salida de la regadera, además de que el tinaco continuamente recibe agua fría conforme se renueva, se infiere que el período de utilización de un tinaco como fuente de agua caliente para el baño se ve muy limitado por diversas variables, como la percepción personal del frio o calor de cada individuo, los hábitos de hora y frecuencia de baño, el perfil de utilización de agua, el arreglo y material de la tubería, entre otros. 


\section{Análisis energético}

\section{Energía almacenada durante el día}

A partir de las temperaturas medias al inicio y final del día $\left(T_{1}\right.$ y $\left.T_{2}\right)$, considerando el inicio cuando se comienza a tener radiación y el final cuando ya no se tiene, se calculó la energía $Q$ acumulada por los depósitos siguiendo la fórmula:

$Q=C_{p} m\left(T_{2}-T_{1}\right)$

donde $C_{p}$ es la constante del calor específico del agua y $m$ es la masa del volumen de agua.

En general, a lo largo del año el depósito de polietileno siempre acumuló mayor calor que el de concreto, con un orden predominante de $1.5-3 \mathrm{kWh}$, mientras que el de concreto se mantuvo entre 1-2 $\mathrm{kWh}$, como se aprecia en la figura 11. Se observa que a valores de irradiación solar menores a $3 \mathrm{kWh} / \mathrm{m}^{2} /$ día hay valores similares de almacenaje de la energía en ambos depósitos, mientras que a mayor energía se tiene mayor almacenaje en el sistema plástico que en el de concreto. Estos resultados coinciden con lo observado en el análisis de temperaturas, donde el depósito de polietileno tiende a contener agua más caliente disponible, lo cual es resultado de ser capaz de almacenar más energía. Igualmente se puede apreciar que, de los dos depósitos, el de concreto tiende a ser más homogéneo en su capacidad de almacenamiento de energía.

\section{Energía durante la noche}

La figura 12 presenta la media, los percentiles 25 y 75 (límites del cuadro) y los extremos de las mediciones de la cantidad de calor acumulado durante el día y el calor disipado durante la noche. Se aprecia que, a lo largo del año, ambos sistemas tienden a disipar calor en magnitudes variables, teniendo un comportamiento que no se mantiene estable, según las condiciones de cada día, lo que refleja la complejidad de sistema. Ambos ganan y disipan más o menos calor en cada día y en cada mes, pero a largo plazo tienden a disipar durante la noche la energía que se acumuló durante el día, lo que se refleja por la temperatura menor y casi igual presentada a las 7:00 horas (mostrada en la figura 9) Igualmente se observa que el depósito de polietileno gana y disipa calor predominantemente entre 1200 y $2400 \mathrm{Wh}$, alcanzado picos en el mes de abril, más que nada por la ausencia de lluvias, mientras que el contenedor de concreto hace lo propio entre 700 y $1800 \mathrm{Wh}$, teniendo durante el año un comportamiento más estable que el depósito de polietileno.

\section{Energía por metro cuadrado}

Como cada tinaco tiene una columna de agua diferente, el área de exposición también es diferente (el tinaco de concreto tiene $2.08 \mathrm{~m}^{2}$ en contacto con la columna de agua, mientras que el de polietileno tiene $1.67 \mathrm{~m}^{2}$ ). La división de la energía absorbida entre el área expuesta permite resultados que no dependen directamente del área. En este caso, como se aprecia en la figura 13, se ha visto que de los dos tinacos, el de polietileno tiende a absorber una mayor cantidad de energía por metro cuadrado de superficie durante el día.

Al presentar estos valores cómo la fracción de irradiación solar absorbida por metro cuadrado respecto la irradiancia recibida (figura 14) se observa un comportamiento con una tendencia homogénea, aunque igualmente se mantiene una mayor absorción de energía en el tinaco de polietileno.

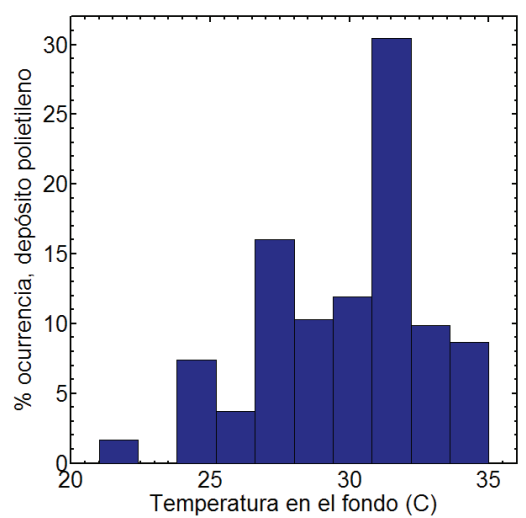

a)

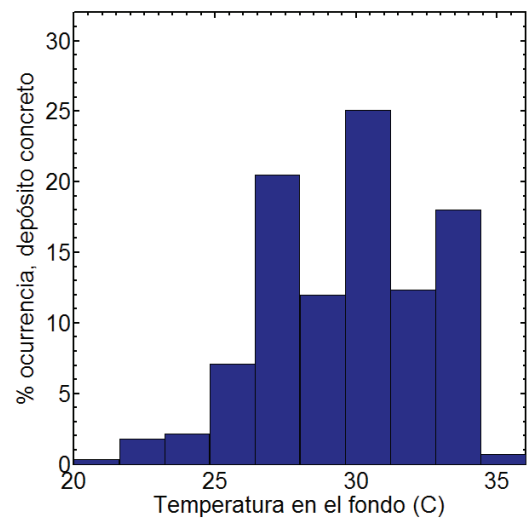

b)
Figura 10. Ocurrencia de temperaturas en el nivel del fondo para depósitos de a) polietileno y b) concreto a las 20:00 horas 

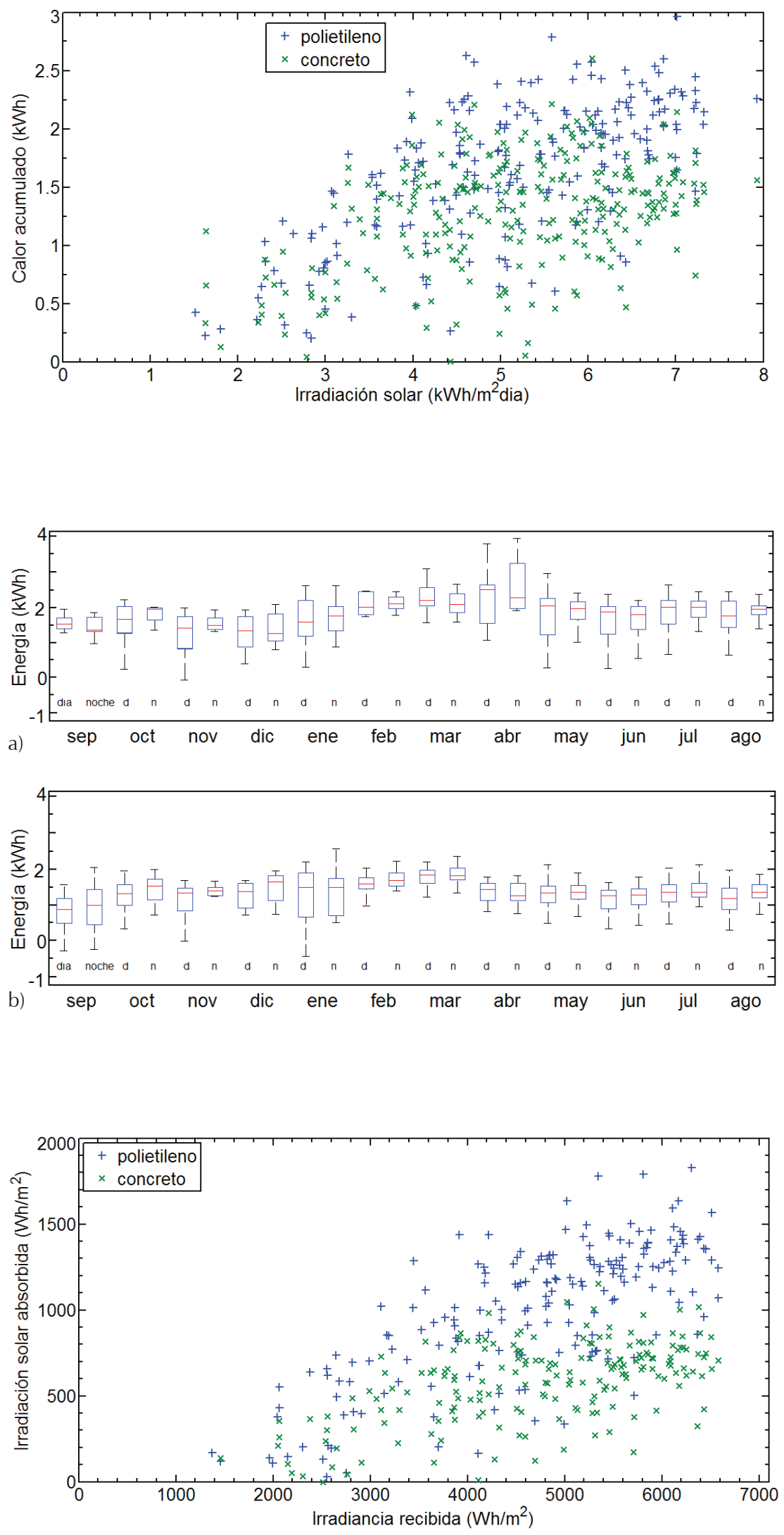

Figura 11. Irradiación solar vs calor acumulado en los contenedores

Figura 12. Calor ganado y calor disipado durante día y noche en los depósitos de a) polietileno y b) concreto
Figura 13. Energía absorbida durante el día por metro cuadrado de superficie en los tinacos 


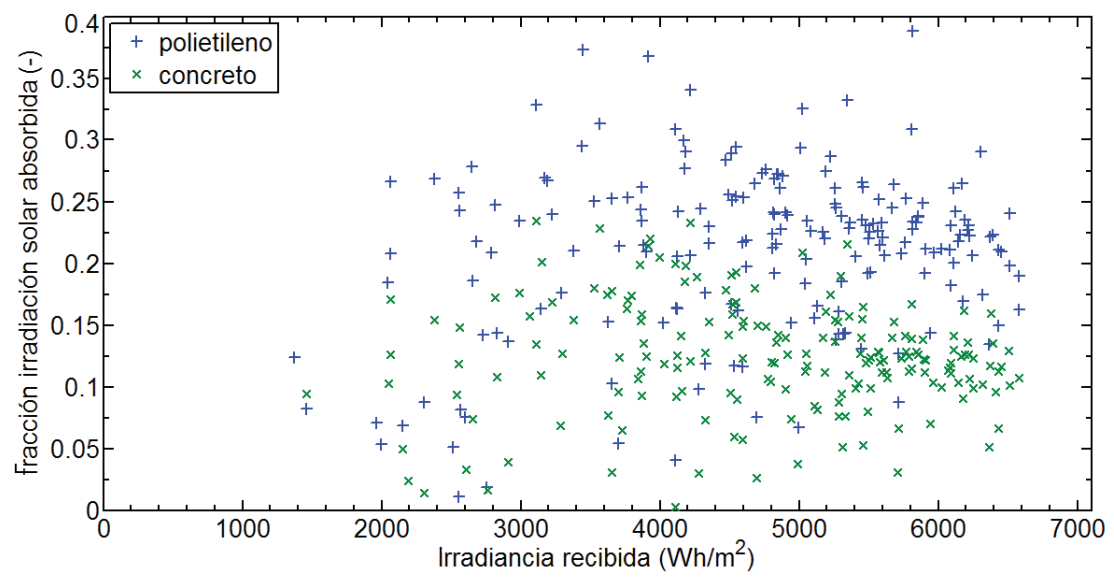

Figura 14. Fracción de energía absorbida durante el día en los tinacos
Dado que el material y los espesores de cada tinaco son diferentes, y en aras de obtener información que explique dicho comportamiento, utilizando las mediciones obtenidas durante la noche se calculó la resistencia térmica de cada tinaco mediante la fórmula.

$R=A\left(T_{a}-T_{t}\right) / Q n$

donde:

$A=$ área expuesta

$T_{a}=$ temperatura del medio ambiente

$T_{t}=$ temperatura del agua en el termotanque $\mathrm{y}$

$Q_{n}=$ calor disipado durante la noche

Se encontró que el tinaco de polietileno, con una resistencia térmica de $0.03 \mathrm{~m}^{2} \mathrm{~K} / \mathrm{W}$ (desviación estándar de 0.004 ), es menos aislante que el de concreto, que tiene una resistencia térmica de $0.05 \mathrm{~m}^{2} \mathrm{~K} / \mathrm{W}$ (desviación estándar de 0.008). Esto explica por qué, al tener una menor resistencia térmica, el tinaco de polietileno es el que tiene mayor acumulación de energía durante el día (figuras 12 a 14) y una cantidad mayor de calor perdido durante la noche (figura 12) y viceversa con el de concreto, aun teniendo una menor área expuesta.

Estos resultados sugieren que los tinacos pueden funcionar como un almacenamiento de energía, pero sólo temporal, pues al no contar con sistemas externos de transferencia de energía ni un aislamiento térmico adecuado, a largo plazo la energía almacenada durante el día se disipa durante la noche, lo que imposibilita tener temperaturas de confort en la mañana que permitan su aprovechamiento, en especial para duchas.

Una temperatura de confort para una ducha se podría obtener en algunos días, en particular en la tarde cuando se ha acumulado el calor en el contenedor, aunque el agua más caliente se encontraría en la parte su- perior del tinaco, donde se tendría que tomar en cuenta la distancia requerida para llegar al punto de suministro de esta agua ( por ejemplo, del tinaco a la regadera). En este estudio no hubo intercambio de agua en los sistemas, cuando se consume por un lado y se completa por medio del flotador. Si se incluye el consumo cotidiano de agua, estas temperaturas podrían bajar debido a la renovación de agua que suele estar más fría que el agua del depósito en uso.

\section{Tinacos de polietileno color negro}

En la región hay indicaciones anecdóticas de que los depósitos de polietileno de color negro, un modelo de uso común en el pasado en la región de Yucatán, generaban temperaturas mucho más elevadas, al grado de volverse normal observar estos contenedores en los techos, pintados de blanco, buscando reducir su temperatura, en especial en los días más calurosos. No se define si esto tuvo alguna influencia en las empresas fabricantes de estos contenedores, pero actualmente se ha cambiado el color y ahora los que se comercializan en la región son tinacos de color beige. Con esta situación en mente se implementó un monitoreo complementario con un tinaco plástico negro, aforado a 360 litros, buscando resaltar alguna diferencia en el comportamiento térmico entre ellos. En este comparativo se posicionó un termopar a una profundidad de $2 / 3$ de la columna de agua para compararlo con su contraparte de contenedor color beige. Como se aprecia en la figura 15, se observó que efectivamente se acumula más calor en un depósito plástico negro, evaluados el mismo día, observándose un incremento de temperatura de $1^{\circ} \mathrm{C}$ aproximadamente en el período más caliente del día, estando las temperaturas igualadas al inicio del día y en ausencia del sol, como se observa en el segmento correspondiente de 0:00 a 6:00 horas. Este resultado es coherente 


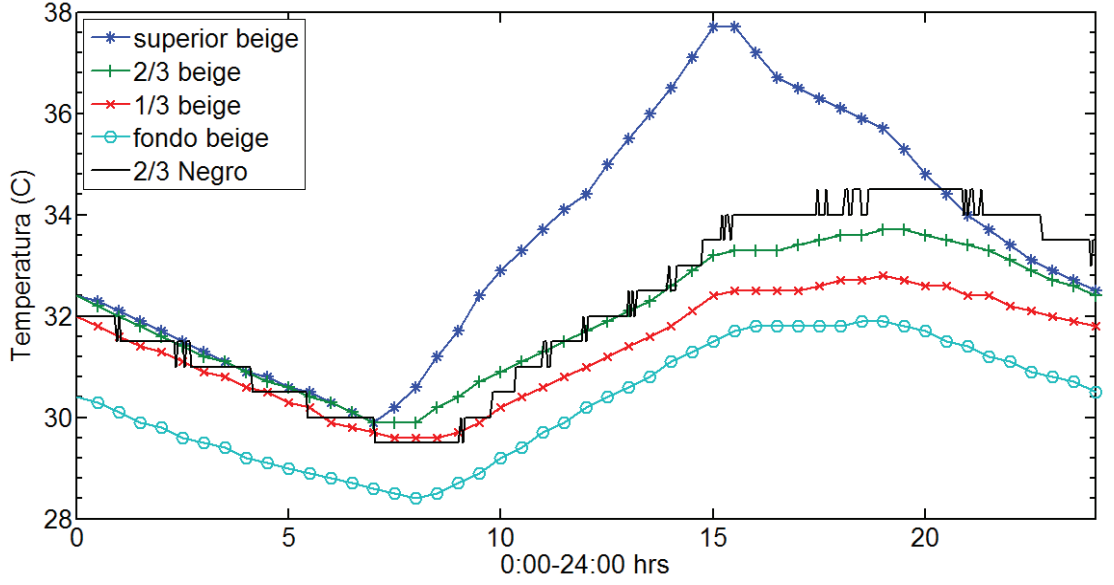

Figura 15.Comparativo de temperaturas de los tinacos plásticos beige y negro aforado a 360 litros con las indicaciones anecdóticas de que un tinaco color negro almacena más calor, pero no se estiman variaciones importantes en el comportamiento que modifiquen los resultados de este estudio.

\section{Conclusiones}

Se llevó a cabo un estudio del comportamiento térmico de dos depósitos de agua construidos con materiales diferentes, uno de polietileno y otro de concreto, obteniéndose mediciones diarias de la temperatura en 4 niveles de altura de la columna de agua de cada uno de ellos.

A partir de los resultados obtenidos se determinó que el depósito de polietileno almacena mayor cantidad de energía que su contraparte de concreto, que se refleja en tener agua alrededor de 4 grados más caliente en la superficie con respecto al de concreto. Sin embargo, en el fondo de los depósitos, la diferencia tiende a ser de 1 o 2 grados en favor del plástico en las horas de mayor temperatura. Igualmente, se determinó que durante la noche se disipa prácticamente todo el calor que fue almacenado durante el día, que sumado al hecho de que la toma de agua de un tinaco es por el fondo, que hay reducción de la temperatura tanto durante la renovación de agua como en el trayecto de la misma del tinaco a la regadera, todo en conjunto, no favorece la utilización de tinacos como fuente de agua caliente por largos períodos. Durante la noche, a las horas en que habitualmente se requiere, se tiene agua que en un momento dado se podría usar para una ducha, aunque depende en gran medida de los hábitos y necesidades de confort del usuario. En las mañanas, debido al calor perdido durante la noche, tendría que descartarse para este fin si se desean temperaturas estándar de confort.

A partir de estos datos se estima que un tinaco convencional no ofrece la posibilidad de utilizarlo como fuente de agua caliente que permita prescindir de algún tipo de calentador, lo cual se encuentra alineado con el hecho que las casas habitación en la región de Yucatán cuenten con un calentador convencional, independientemente del calor habitual de la región.

\section{Agradecimientos}

Se agradece el trabajo efectuado por Ricardo Gamboa y Diego Medina para la implementación del sistema experimental, y el efectuado por Juan Enrique Vera para la digitalización de la información.

\section{Referencias}

Barron J.E. Estudio comparativo de dos sistemas de calentadores solares en una zona cálida-subhúmeda (maestría en ingeniería), Mérida, Centro de Investigación Científica de Yucatán, 2012.

Boduch M. y Fincher W. Standards of human comfort: relative and absolute, The University of Texas at Austin, School of architecture, Meadows seminar, Fall, 2009.

Diario Oficial de la Federación. Calentadores instantáneos de agua para uso doméstico- Gas natural o L.P. Norma oficial mexicana NOM-022-SCFI-1993. Fecha de publicación: 14 de octubre de 1993, fecha de modificación: 18 de junio de 2001.

Emery A.F. y Kippenhan C.J. A long term study of residential home heating consumption and the effect of occupant behavior on homes in the Pacific Northwest constructed according to improved thermal standards, Special Issue from The Second ASME-ZSIS International Thermal Science Seminar (ITSS II), Bled, Slovenia, 13-16 de junio de 2004, volumen 31 (número 5), pp. 677-693.

Gagge A.P., Stolwijk J.A.J., Hardy J.D. Comfort and thermal sensations and associated physiological responses at various ambient temperatures. Environmental Research, volumen 1 (número 1), junio de 1967: 1-20. 
Guerra S.O., Itard L., Visscher H. The effect of occupancy and building characteristics on energy use for space and water heating in Dutch residential stock. Energy and Buildings, volumen 41, 2009: 1223-1232.

Instituto Nacional de Estadística y Geografía (INEGI). Principales resultados del censo de población y vivienda 2010, México, 2010.

Kordana S., Słyś D., Dziopak J. Rationalization of water and energy consumption in shower systems of single-family dwelling houses. Journal of Cleaner Production, volume 82 (número 1), noviembre 2014: 58-69.

Thermostatic Mixing Valve Manufacturers Association (TMVMA). Recommended Code of Practice for Safe Water Temperatures, Núm. 1, marzo de 2000.

\section{Este artículo se cita: \\ Citación estilo Chicago}

Diego-Ayala, Ulises, José Gonzalo Carrillo-Baeza. Estudio del comportamiento térmico de agua para uso residencial en tinacos de concreto y polietileno en un clima cálido-subhúmedo. Ingeniería Investigación y Tecnología, XVI, 04 (2015): 573-583.

\section{Citación estilo ISO 690}

Diego-Ayala U., Carrillo-Baeza J.G. Estudio del comportamiento térmico de agua para uso residencial en tinacos de concreto y polietileno en un clima cálido-subhúmedo. Ingeniería Investigación y Tecnología, volumen XVI (número 4), octubre-diciembre 2015: 573-583.

\section{Semblanzas de los autores}

Ulises Diego-Ayala. Doctor en ingeniería mecánica, especializado en análisis de energía. Miembro del SNI, ingeniero mecánico por el Instituto Tecnológico de Mérida con maestría por el Centro Nacional de Investigación y Desarrollo Tecnológico y doctorado por el Imperial College (Londres, Inglaterra). Previo al doctorado trabajó en el Instituto Mexicano del Petróleo y en empresas colaboradoras de PEMEX en proyectos de construcción y mantenimiento. Posterior al doctorado trabajó en Barcelona en el desarrollo de propuestas para trabajos de investigación de la Comunidad Europea y en proyectos de desarrollo de transmisiones de variación continua (CVT) y sistemas mecatrónicos. Actualmente colabora en el CICY en proyectos de energía renovable.

José Gonzalo Carrillo-Baeza. Doctor en ciencia de materiales. Profesor investigador del Centro de Investigación Científica de Yucatán desde el 2008, ingeniero mecánico, doctorado por la Universidad de Liverpool, Inglaterra en materiales compuesto avanzados, miembro continuo del SNI 2008-2015, nivel 1, más de 50 artículos arbitrados y en extenso, internacionales y nacionales publicados, dos patentes en proceso, dirección de tesis de 30 estudiantes de licenciatura y posgrado graduados, docente en el Posgrado de Materiales Poliméricos y Posgrado en energía renovable del CICY, dos proyectos externos vigentes como responsable técnico y dos más como participante. 